\title{
Guidelines for Adapting Light Microscopy Deconvolution Methods to 3D Biological STEM
}

\author{
B.M. Northan*, R. Sougrat**, and N. de Jonge*** \\ * Media Cybernetics, 4340 East-West Highway, \#400, Bethesda, MD 20814 \\ ** Cell Biology and Metabolism Branch, NCICHD, National Institute of Health, 18 Library Drive, \\ Bethesda, MD 20892-5430 \\ *** Molecular Physiology and Biophysics, Vanderbilt University Medical Center, 2215 Garland \\ Ave, Nashville TN 37232-0615 and Materials Science and Technology Division, Oak Ridge \\ National Laboratory, 1 Bethel Valley Rd., Oak Ridge, TN 37831-6064
}

Advances in spherical aberration correction of the scanning transmission electron microscope (STEM) have led to a reduced depth of field of the STEM probe [1]. This has made it possible to form a 3D dataset by the stacking of 2D images collected at a sequence of depth intervals through the sample thickness. Each 2D image contains the signal from objects at its corresponding depth within the sample and also the out-of-focus signals from the adjacent images (above and below). A similar effect occurs in widefield fluorescence light microscopy, where the effect is corrected for by applying a deconvolution algorithm, of which several variants exist. We discuss modification of the Expectation-Maximization deconvolution procedure originally developed for light microscopy [2], and its subsequent application to 3D STEM.

In widefield light microscopy the emission and detection of photons can be modeled using Poisson statistics. The Expectation-Maximization algorithm maximizes the likelihood function of the restored image from the acquired image, given a known point spread function (PSF), and assuming Poisson emission and detection statistics. The algorithm can also be modified to estimate the PSF along with the restored image (blind deconvolution) [2]. Several constraints are used to ensure the algorithm converges to a realistic solution: the restored image is forced to be non-negative; an hourglass spatial constraint based on system optics is applied to the PSF in the spatial domain; the frequency response of a diffraction-limited system is zero outside of radial and axial bandlimits and within a biconic missing cone region [3]. Thus, a bandlimit and missing cone constraint are applied to the PSF in the frequency domain. Since 3D STEM is similar to 3D widefield light microscopy [4] a similar set of constraints can be used.

For 3D STEM, there are several additional factors that need to be taken into account. For the typical magnifications used to image a biological sample, the sample is under-sampled in the lateral direction since the resolution of an aberration corrected STEM is $<0.1 \mathrm{~nm}$, i.e., the pixel size is larger than the probe size [4]. This means that each pixel in a vertical line receives the same number of electrons until the beam becomes larger than a pixel and the effective axial resolution is reduced by the quotient of the pixel size and the probe size. The beam opening angles commonly used are only several tens of milliradians, so the missing cone region in the frequency domain and the axial extent of the PSF are both large. Furthermore, chromatic aberration and beam energy-spread will also reduce the axial resolution [5]. Finally, scan distortions will occur when changing the focus position away from the eucentric height. We examined application of the existing deconvolution algorithm taking each of these factors into account. 
The performance of the deconvolution algorithm was evaluated using conventional thin sections containing biological specimens stained by metal particles [4]. The presence of individual grains of staining of known object shape (and additionally, gold particles of known shape) made it possible to solve for the PSF. We extracted a measured PSF from objects of known size and shape from a small sub-region of the acquired image and used this measured PSF to deconvolve the entire image. A comparison between three variations of the algorithm was performed, i.e., deconvolution using the measured PSF, deconvolution using a theoretically generated PSF, and blind deconvolution. To illustrate the deconvolution algorithm applied to 3D STEM, the results of applying the blind algorithm to a 3D image data-set is shown in Fig. 1. The data was recorded with an aberrationcorrected STEM at $200 \mathrm{kV}$ (JEOL 2200FS STEM/TEM equipped with a CEOS aberration corrector) from a position where the sample has a bundle of microtubules and gold particles at different depths.

\section{References}

[1] K. van Benthem, et al., Appl. Phys. Lett. 87 (2005) 034104

[2] T. J. Holmes, J. Opt. Soc. Am. 9 (1992) 1052

[3] T. J. Holmes et al., Handbook of Biological Confocal Microscopy, 1995, Plenum Press: New York. 389

[4] N. de Jonge, et al., Nanotechnology in Biology and Medicine-Methods, Devices and Application, 2007, CRC Press: Boca Raton. 13.1

[5] A. Y. Borisevich, A. R. Lupini, S. J. Pennycook, PNAS, 103 (2006) 3044

[6] We are grateful to L.F. Allard, D. Blom, D.T. Burnette, J.F. Deatherage, J. LippincottSchwartz, A.R. Lupini, S.J. Pennycook, D.W. Piston, and J.R. Price. This work was supported by the Laboratory Directed R\&D Program of Oak Ridge National Laboratory (ORNL), ORNL's High Temperature Materials Laboratory User Program, sponsored by the U. S. Department of Energy, Office of Energy Efficiency and Renewable Energy, Vehicle Technologies Program, Vanderbilt University Medical Center, NIH grant R01GM081801 (NJ), Intramural Program of NICHD (RS).
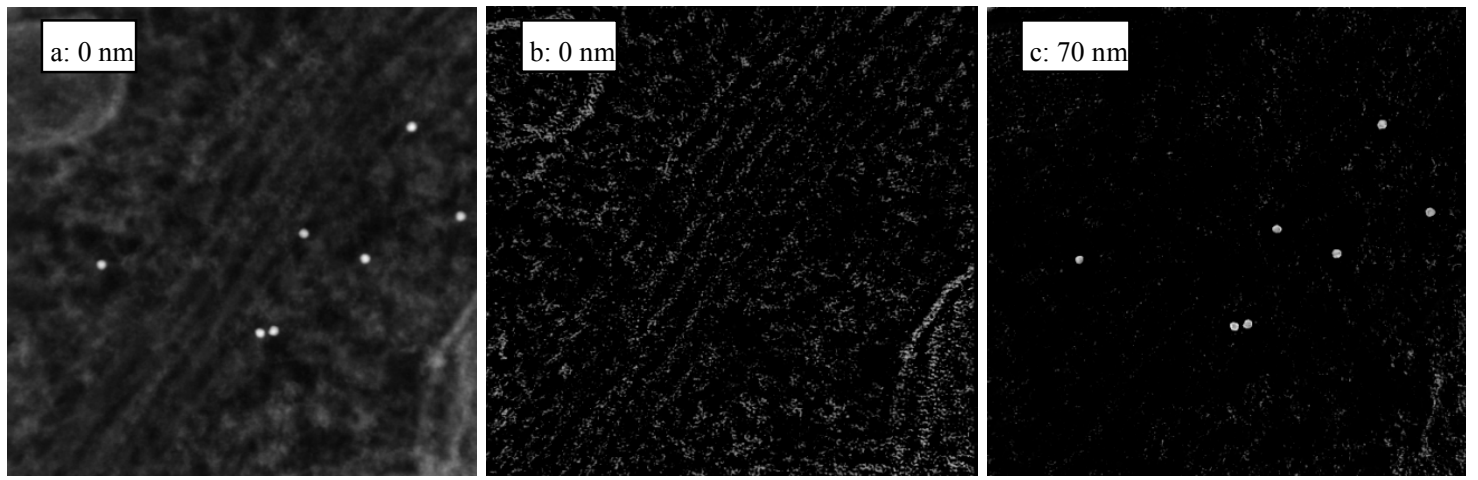

FIG. 1. a) A slice of a 3D STEM data set containing microtubules also contains out of focus signal from gold particles. b) After deconvolution of the out-of-focus signal from the gold particles is not present in the plane containing microtubules. c) Similarly after deconvolution the plane containing gold particles (varying $70 \mathrm{~nm}$ in focus) does not show strong signal from the microtubules. 